\title{
Eventos de vida produtores de estresse e transtornos mentais comuns: resultados do Estudo Pró-Saúde
}

\author{
Stressful life events and common mental \\ disorders: results of the Pro-Saude Study
}

Claudia S. Lopes 1

Eduardo Faerstein 1

Dóra Chor 2

\footnotetext{
1 Instituto de Medicina Social, Universidade do Estado do Rio de Janeiro. Rua São Francisco Xavier 524, 7o andar, Rio de Janeiro, RJ 20559-900, Brasil. Iopes@uerj.br 2 Departamento de Epidemiol ogia e Métodos Quantitativos em Saúde, Escola Nacional de Saúde Pública, Fundação Oswaldo Cruz. Rua Leopoldo Bulhões 1480, Rio de Janeiro, RJ 21041-210, Brasil.
}

\begin{abstract}
This study investigates the association between stressful life events (SLE) and common mental disorders (CMD) among university empl oyees in Rio de Janeiro, Brazil. Phase 1 sectional data from a cohort study (the Pro-Saude Study) were collected among 4,030 participants. A standardized self-administered questionnaire (General Health Questionnaire) assessed the presence of CMD during the previous two weeks, the occurrence of SLE in the previous 12 months, and socio-demographic characteristics. After adjustment for gender, age, income, and marital status, severe financial problems were associated with the greatest risk of CMD (OR $=2.6 ; 95 \% \mathrm{Cl}: 2.2$ 3.0), followed by physical aggression ( $\mathrm{OR}=2.1 ; 95 \% \mathrm{Cl}$ : $1.5-2.8)$, disruption of a love relationship $(O R=1.9 ; 95 \% \mathrm{Cl}: 1.6-2.3)$, forced change of residence $(\mathrm{OR}=1.9 ; 95 \% \mathrm{Cl}: 1.5-2.4)$, serious disease $(\mathrm{OR}=1.8 ; 95 \% \mathrm{Cl}: 1.5-2.1)$, hold-up or theft $(\mathrm{OR}=1.5 ; 95 \% \mathrm{Cl}: 1.2-1.8)$, and hospitalization $(\mathrm{OR}=$ $1.4 ; 95 \% \mathrm{Cl}: 1.1-1.8)$. Results suggest that long-term effects of SLE should be investigated, as well as other potential modifying factors in the development and maintenance of CMD in order to support more effective mental health interventions.
\end{abstract}

Key words Stressful Events; Mental Disorders; Cohort Studies; Cross-Sectional Studies

Resumo O objetivo do estudo é investigar a associação entre eventos de vida produtores de estresse (EVPE) e transtornos mentais comuns (TMC). Dados seccionais da fase 1 de um estudo de coorte (Estudo Pró-Saúde) foram col etados entre 4.030 funcionários. I nformações foram obti das por meio de um questionário multidimensional autopreenchível (General Health Questionnaire) que avaliou a presença de TMC nas duas semanas anteriores, ocorrência de EVPE nos 12 meses anteriores e variáveis soci odemográficas. Após ajuste por sexo, idade, renda e si tuação conjugal, problemas financei ros graves foram os eventos associados a uma chance mais el evada de TMC (OR =2,6; IC95\%: 2,2-3,0), seguidos de agressão física (OR =2,1; IC95\%: 1,5-2,9), mudança forçada de moradia (OR =1,9; IC95\%: 1,5-2,4), doença grave (OR =1,8; IC95\%: 1,5-2,1), rompi mento de relação amorosa (OR =1,9; IC95\%: 1,6-2,3), internação hospitalar (OR =1,4; IC95\%: 1,1-1,8) e assal to ou roubo (OR =1,5; IC95\%: 1,2-1,8). Os resultados apontam para a necessi dade de se investi gar efei tos de longo prazo associados a estes eventos, assi m como de fatores que potencialmente modifiquem seu impacto, de forma a subsidiar ações de prevenção mais efetivas na área da saúde mental.

Palavras-chave Eventos Estressantes; Transtornos Mentais; Estudo de Coortes; Estudos Transversais 


\section{Intro dução}

Os principais estudos que avaliaram a saúde mental de indivíduos na comunidade têm demonstrado que cerca de $90 \%$ da morbidade psiquiátrica, nessas populações, compõe-se de distúrbios não-psicóticos. Os principais distúrbios evidenciados nestes estudos têm sido depressão e ansiedade, incluindo uma série de queixas inespecíficas e somáticas (Bebbington et al., 1981; Cheng, 1988, 1989; Coutinho, 1995; Finlay-Jones \& Burvill, 1977; Goldberg \& Huxley, 1992).

Estudos conduzidos em populações ocidentais têm mostrado que as prevalências de transtornos mentais não-psicóticos variaram de $7 \%$ a $26 \%$, com uma média de $17 \%$ ( $12,5 \%$ em homens e $20 \%$ em mulheres). U m outro achado importante é que os quadros depressivos têm representado o terceiro problema de saúde em mulheres nos países desenvolvidos e o quinto em países subdesenvolvidos, depois de causas maternas e de al gumas doenças transmissíveis. Estes transtornos contribuem ainda para um terço dos dias perdidos por doença no trabalho e um quinto de todas as consultas de atenção primária. Pacientes com transtornos mentais menores apresentam também taxas de mortalidade mais elevadas e prejuízos importantes nas funções social e física (Bhugra, 1993; Coutinho, 1995).

No Brasil, estudos conduzidos em clientelas de serviços de saúde em São Paulo e Porto Alegre mostraram que cerca de $50 \%$ dos pacientes atendidos apresentavam algum distúrbio mental não-psicótico (Busnello et al., 1983; Iacoponi, 1989; Mari, 1986, 1987). Mais recentemente, Villano (1998) observou a presença desses distúrbios em cerca de um terço dos pacientes de um ambulatório de atendimento clínico em um hospital universitário, no Rio de Janeiro.

Nas últimas décadas, os estudos epidemiológicos têm contribuído de forma significativa para uma mel hor compreensão dos elos entre fatores do ambiente social e a origem e o curso de distúrbios psiquiátricos. Exemplos desses estudos são o International Pi lot Study in Schizophrenia (WHO, 1979) e Determinants of Outcome Project (Sartorius et al., 1986). Grande parte desta produção acadêmica está voltada para o papel dos chamados eventos de vida produtores de estresse (stressful life events) como fatores de risco para transtornos mentais nãopsicóticos (Brown \& Harris, 1978; Costello, 1982; Eaton, 1978; Finlay-Jones \& Burvill, 1977; Kendler et al., 1998; Paykel, 1978; Surtees et al., 1986).
Talvez a maior dificuldade em se avaliar o papel dos determinantes sociais na iniciação e curso dos distúrbios psiquiátricos repouse na possibilidade de causalidade bidirecional associada a estes eventos. Fatores ambientais desfavoráveis estão sabidamente associados ao aparecimento e/ ou à maior severidade no curso de grande parte dos distúrbios psiquiátricos. Por outro lado, a doença mental pode provocar uma série de prejuízos sociais na vida do indivíduo, como baixo nível educacional, desemprego, ruptura de relações pessoais, etc. Portanto, distinguir fatores de risco de efeitos adversos constitui um dos grandes desafios da área.

$\mathrm{Na}$ América Latina, e no Brasil em particular, existem uma série de fatores que contribuem para a elevada prevalência de eventos de vida estressantes e de transtornos mentais na população, tais como processos acelerados de urbanização e industrialização, acesso desigual aos cuidados de saúde, condições de moradia inadequadas, distribuição desigual da renda, desemprego, altas taxas de violência e criminalidade. Em Pelotas, Lima et al., (1996), observaram uma prevalência de transtornos mentais comuns (TMC) de $22,7 \%$, que aumentava com a idade. Nessa população, a presença de eventos de vida produtores de estresse (EVPE), com exceção de migração e acidentes, estava positivamente associada aos TMC, e a probabilidade de TMC aumentou, linearmente, com o número de eventos desfavoráveis.

Considera-se, portanto, que a relevância sociológica e epidemiológica do tema e a pequena produção empírica nacional a respeito tornam pertinente a investigação da associação entre a ocorrência de EVPE eTMC, em uma população adulta no contexto urbano brasileiro. Por um lado, a realização de estudos desse tipo, voltados para a exploração de determinantes de natureza social e psicológica nas grandes metrópoles adquire importância estratégi$c a$, porque se trata do ambiente circundante a que estão submetidas parcelas crescentes da população brasileira. Por outro, além de custos el evados, as características da vida urbana em cidades como o Rio de Janeiro impõem sérias dificuldades operacionais para a realização de pesquisas de campo na população geral. Como vem se revelando especial mente difícil conseguir a adesão de potenciais participantes em seus domicílios, a abordagem de populações trabal hadoras em seus locais de trabalho constitui uma alternativa. Neste quadro, a população de funcionários de uma universidade oferece, em decorrência do tamanho amostral, a possibilidade de maior poder estatístico nas análises realizadas e, em decorrência da faci- 
lidade de acesso, maior operacionalidade na coleta de dados. Tais características favorecem a realização de um estudo com os objetivos propostos.

O objetivo deste estudo é avaliar a associação entre a ocorrência de eventos de vida produtores de estresse e a presença de transtornos mentais comuns entre funcionários técnicoadministrativos de uma universidade no Estado do Rio de Janeiro.

\section{Material e métodos}

Delineamento do estudo

e população de referência

Trata-se de um estudo seccional, cujos dados coletados em 1999 constituem a "linha de base" (Fase 1) do Estudo Pró-Saúde. O Estudo PróSaúde constitui um estudo de coorte entre os funcionários técnico-administrativos do quadro efetivo de uma universidade pública no Estado do Rio de Janeiro. Foram excluídos funcionários aposentados, cedidos a outra instituição e licenciados sem vencimentos.

\section{Instrumentos}

- Características gerais do questionário aplicado

O instrumento utilizado na Fase 1 consistiu de questionário autopreenchível, aplicado em grupos com apoio de pessoal treinado. O questionário contemplou, entre outros aspectos, a história e situação atual das condições sócioeconômicas, padrões de dieta, atividade física, consumo de tabaco (ativo e passivo) e álcool; história de diagnósticos e tratamentos médicos; consumo de medicamentos, e uso de terapias não-convencionais; práticas de prevenção e diagnóstico precoce; e outros comportamentos e exposições com repercussões sobre a saúde. Participantes do sexo feminino foram solicitadas a fornecer informações adicionais em relação à vida reprodutiva e a condições de saúde específicas.

Blocos específicos de perguntas foram incluídos para a aferição de eventos de vida produtores de estresse e transtornos mentais comuns.

a) Eventos de vida produtores de estresse: para a avaliação dos EVPE, optou-se pelo uso de perguntas simples e breves, de forma a se evitar o cansaço dos respondentes e possíveis viéses de informação. Os eventos cobertos foram: episódio grave de doença; falecimento de pa- rentes próximos; internação hospitalar; separação/divórcio; mudança forçada de moradia; problemas financeiros mais graves que os habituais; agressão física; e assalto ou roubo com violência. O período de referência para a ocorrência de eventos foram os 12 meses anteriores ao preenchimento do questionário, de acordo com o recomendado na literatura (Brown \& Harris, 1978; Brugha et al., 1990; Cooke, 1986; Costello, 1982; Kendler et al., 1995).

b) Transtornos mentais comuns: o componente referente à presença de TMC foi avaliado por meio da aplicação do GHQ-12 (General Health Questionnaire) (Goldberg \& Blackwell, 1972), um instrumento de rastreamento para transtornos psiquiátricos menores (depressão e ansiedade) já bem estabelecido e amplamente utilizado em pesquisas internacionais e nacionais nesta área (Araya et al., 1992; Mari \& Williams, 1985; Stanfeld et al., 1997; Weich \& Lewis, 1998). Esse questionário autopreenchível foi validado na sua versão original (Stanfeld \& Marmot, 1992) e na sua versão brasileira (Mari $\&$ Williams, 1985), tendo, em ambos os casos, o Clinical Interview Schedule (Goldberg \& Blackwell, 1970), como padrão ouro. O ponto de corte utilizado para o questionário considerou cada item como ausente ou presente (0 ou 1), de acordo com o método do General Health Questionnaire (Goldberg \& Williams, 1988; Weich \& Lewis, 1998). Aqueles que foram positivos para 3 itens do GHQ (em 12 itens) foram classificados como casos de TMC (Goldberg \& Williams, 1988). O período de referência do GHQ foram as duas semanas anteriores ao preenchimento do questionário.

- Pré-testes, estudo piloto

e estudo de confiabilidade

Cinco etapas de pré-testes foram realizadas, envolvendo cerca de 150 voluntários com perfil social e funcional semelhante ao da população-alvo. Assim, funcionários técnico-administrativos de três instituições públicas diferentes preencheram quatro versões de todo o questionário. Após a aplicação desses pré-testes, foram analisadas a compreensão das perguntas formuladas, e também a distribuição das respostas por categorias de sexo e escolaridade (Faerstein et al., 1999).

O estudo piloto foi realizado em julho de 1999, em uma amostra sistemática de duzentos funcionários administrativos contratados, mas não pertencentes ao quadro efetivo da Universidade, por apresentarem perfis social e funcional semelhantes aos dos participantes do estudo principal. A composição desta amos- 
tra procurou aproximar-se o mais possível das características sócio-demográficas dos funcionários efetivos da universidade, principalmente no que diz respeito ao gênero, faixa etária e escolaridade.

O estudo de confiabilidade (teste- reteste) foi realizado com os mesmos funcionários (192 que responderam ao questionário na segunda aferição), duas semanas após a primeira.

A avaliação da confiabilidade teste-reteste da informação fornecida pelos respondentes em relação aos EVPE foi realizada por intermédio do coeficiente kappa (k) (Fleiss, 1981). Para efeito de interpretação, utilizou-se os critérios sugeridos por Landis \& Koch (1977), no qual se propõe cinco categorias para as estimativas de confiabilidade com base nos valores encontrados para o kappa: excelente $(>0,80)$; muito boa $(0,61$ a 0,80$)$; boa $(0,41$ a 0,60$)$; regular $(0,21$ a $0,40)$; fraca $(0,01$ a 0,20$)$ e pobre $(0,00)$. De acordo com os resultados encontrados, a confiabilidade foi considerada "muito boa" para quase todos os eventos. Foi encontrada uma confiabilidade "excelente" para a questão referente à internação hospitalar em decorrência de doença ou acidente. A questão referente à ocorrência de problema de saúde grave obteve uma confiabilidade considerada boa. A única questão cuja confiabilidade obtida foi regular, foi aquela referente ao fato do indivíduo ter sofrido uma agressão física (Lopes \& Faerstein, 2001).

- Enumeração da população-alvo e coleta de dados

A enumeração da população-alvo foi realizada com base em banco de dados fornecido pela Superintendência de Recursos Humanos da universidade, listagens fornecidas pelo órgão responsável pela elaboração da folha de pagamentos e, adicionalmente, listagens fornecidas pelas diversas unidades e setores. Com a combinação dessas três fontes de informação, a universidade foi mapeada e setorizada de acordo com a conveniência operacional do trabaIho de campo, em conjunto com a confirmação da lotação de cada funcionário, e o início da sensibilização dos potenciais participantes.

A coleta de dados foi conduzida entre agosto e outubro de 1999, em auditórios de cada uma das unidades da universidade, durante o horário de expediente, após intenso trabalho de divulgação. Peso e estatura foram aferidos em todos os participantes.

\section{- Aspectos éticos}

Antes da coleta de dados, os protocolos referentes à pesquisa foram submetidos aos Comitês de Ética da universidade e do hospital universitário. Em ambos os comitês, a pesquisa foi considerada adequada para ser realizada em população humana.

A adesão ao seguimento foi voluntária, esclarecendo-se aos funcionários que nenhum tipo de penalidade seria aplicada aos que não desejassem participar. Após esclarecimentos sobre a pesquisa, foi solicitada leitura e assinatura de Termo de Consentimento.

Durante a coleta de dados, os questionários foram identificados por meio de numeração cuja ligação ao nome do funcionário é de conhecimento exclusivo da coordenação da pesquisa. Os dados foram analisados somente de forma agregada, garantindo-se que os nomes dos participantes, bem como sua associação com qualquer informação coletada jamais farão parte de relatórios ou artigos científicos que venham a ser divulgados.

\section{- Análise dos dados}

Todas as questões referentes à experiência prévia de EVPE e à presença de transtornos mentais comuns foram avaliadas como variáveis dicotômicas (sim/não). As relações entre EVPE e TMC foram examinadas por meio de regressão logística não-condicional (backward selection), com ajuste pelas variáveis sexo, idade, renda, situação conjugal e escolaridade. O efeito dos EVPE no status de caso de TMC foi expresso por intermédio das odds ratios (OR) e seus respectivos intervalos de 95\% de confiança (IC 95\%). As análises foram realizadas usando-se o programa estatístico Stata.

\section{Resultados}

A população do estudo constou de 4.030 funcionários, que representaram $90,6 \%$ da população de 4.448 funcionários técnico-administrativos da universidade elegíveis para o estudo.

Cerca de $70 \%$ dos funcionários têm entre 25 e 44 anos de idade, e pouco mais da metade (55\%) são do sexo feminino (Tabela 1 ). Há funcionários nos diversos níveis de escolaridade, mas a grande maioria possui o segundo ou o terceiro graus completos (76\%). Sessenta e um por cento dos funcionários são casados ou vivem em união e cerca de $74 \%$ possuem uma

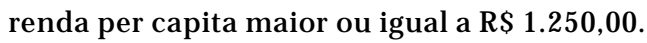


Com relação ao relato de eventos de vida produtores de estresse (EVPE) nos 12 meses anteriores ao preenchimento do questionário, “ter passado por dificuldades financei ras graves" foi o evento mais freqüente, tendo sido relatado por cerca de metade dos funcionários. Os outros eventos apresentaram freqüências menores, com relato de "problemas sérios de saúde" por $22,4 \%$, "rompimento de relação amorosa" por $16,6 \%$, "morte de parente próximo" por $12,7 \%$, ter sido "vítima de assalto ou roubo" por $11,5 \%$, "mudança forçada de moradia" por 8,7\%, "internação hospitalar por pelo menos uma noite" por 7,5\% e "ter sido vítima deagressão física" por 4,1\% (Tabela 2).

A prevalência de transtornos mentais comuns, segundo o ponto de corte adotado, foi de $29 \%$ (22,2\% dos homens e $34,4 \%$ das mulheres). Quando avaliamos a associação entre a presença de TMC e as características sócio-demográficas da população, observamos que apenas sexo ( $p<0,001)$, renda $(p=0,001)$ e situação conjugal $(p=0,03)$ apresentaram associação estatisticamente significativa com TMC. A idade dos indivíduos ( $p=0,506)$ e a escolaridade $(p=0,972)$ não apresentaram associação estatisticamente significativa com TMC nesta população.

A avaliação da associação entre ocorrência de EVPE e TMC nesta população mostrou que, à exceção de morte de parente próximo, todos os outros eventos de vida se associaram de forma estatisticamente significante com a presença de TMC (Tabela 3). Após análise de regressão logística e ajuste pelas variáveis sócio-demográficas investigadas, observou-se que ter passado por dificuldades financeiras graves (OR =2,60; IC95\%: 2,24-3,02), ter sido vítima de agressão física (OR =2,13; IC95\%: 1,54-2,95), rompimento de relação amorosa $(O R=1,94)$, mudança forçada de moradia (OR =1,90; IC95\%: $1,50-2,40)$ e problema sério de saúde ( $O R=1,81$; IC95\%: 1,54-2,95) foram os eventos de vida mais fortemente associados à presença de TM C nesta população (Tabela 4).

\section{Discussão}

Os resultados deste estudo mostram associação entre eventos de vida produtores de estresse (ocorridos nos 12 meses anteriores à pesquisa) e a presença de transtornos mentais comuns. Estes achados estão de acordo com estudos prévios conduzidos no Brasil e em outros países. Ter passado por dificuldades financei ras graves foi o evento mais fortemente associado com TMC $(O R=2,60)$. Este achado é consistente com o estudo conduzido por Weich \& Lewis
Tabela 1

Características sóciodemográficas dos funcionários técnico-administrativos de uma universidade no Estado do Rio de J aneiro $(n=4.030)$. Estudo Pró-Saúde, 1999.

\begin{tabular}{|c|c|c|}
\hline Característica & $\mathrm{n}$ & $\%$ \\
\hline \multicolumn{3}{|l|}{ Sexo } \\
\hline Feminino & 2.240 & 55,58 \\
\hline Masculino & 1.790 & 44,42 \\
\hline \multicolumn{3}{|l|}{ Idade (anos) } \\
\hline$\leq 24$ & 46 & 1,14 \\
\hline $25-34$ & 1.078 & 26,75 \\
\hline $35-44$ & 1.740 & 43,18 \\
\hline $45-54$ & 886 & 21,99 \\
\hline$\geq 55$ & 280 & 6,95 \\
\hline \multicolumn{3}{|l|}{ Escolaridade } \\
\hline 1o grau incompleto & 276 & 6,85 \\
\hline 10 grau completo & 671 & 16,81 \\
\hline 2o grau completo & 1.429 & 35,80 \\
\hline 3 o grau completo & 1.616 & 40,48 \\
\hline \multicolumn{3}{|l|}{ Situação conjugal } \\
\hline Casado/vive em união & 2.371 & 60,97 \\
\hline Solteiro/viúvo/divorciado & 1.518 & 39,03 \\
\hline \multicolumn{3}{|l|}{ Renda per capita (R\$) } \\
\hline$>500<1.250$ & 1.037 & 25,73 \\
\hline$>1.250<2.750$ & 1.391 & 34,52 \\
\hline$>2.750$ & 1.602 & 39,75 \\
\hline
\end{tabular}

Tabela 2

Freqüência de eventos de vida produtores de estresse (EVPE) nos 12 meses anteriores ao preenchimento do questionário, entre funcionários técnico-administrativos de uma universidade no Estado do Rio de J aneiro ( $n=4.030)$. Estudo Pró-Saúde, 1999.

\begin{tabular}{lrr}
\hline EVPE & $\mathrm{n}$ & $\%$ \\
\hline Problema grave de saúde & 896 & 22,39 \\
Internação hospitalar & 298 & 7,47 \\
Dificuldades financeiras graves & 1.998 & 49,84 \\
Morte de parente próximo & 505 & 12,65 \\
Mudança forçada de moradia & 347 & 8,66 \\
Rompimento de relação amorosa & 665 & 16,64 \\
Vítima de assalto ou roubo & 459 & 11,45 \\
Vítima de agressão física & 164 & 4,09 \\
\hline
\end{tabular}


(1998), em um estudo de coorte de base popuIacional na Grã-Bretanha, que mostrou ser o "estresse financeiro" o evento de vida mais fortemente associado com TMC, segundo os critérios do GHQ-12, nesta população. Outros eventos, como "ter si do vítima de agressão física" $(O R=2,13)$, "rompimento de rel ação amorosa" (OR =1,94), "mudança forçada de moradia" (OR $=1,90)$ e "problema sério de saúde" ( $O R=1,81)$ também estiveram positivamente associados com TMC e achados semelhantes são descritos nas literaturas nacional e internacional (Finlay-Jones \& Brown, 1981; Horowitz et al., 1977; Lima et al., 1996).

A principal limitação deste estudo decorre da utilização de dados seccionais, que não per-

Tabela 3

Prevalência de transtornos mentais comuns (TMC), segundo a ocorrência de eventos de vida produtores de estresse (EVPE), entre funcionários técnico-administrativos de uma universidade no Estado do Rio de J aneiro. Estudo Pró-Saúde, 1999.

\begin{tabular}{lrrr}
\hline EVPE & \multicolumn{3}{c}{ TMC } \\
& $n$ & $\%$ & $p$ \\
\hline Problema grave de saúde & 352 & 39,3 & $<0,001$ \\
Internação hospitalar & 109 & 36,6 & 0,003 \\
Dificuldades financeiras graves & 774 & 38,7 & $<0,001$ \\
Morte de parente próximo & 157 & 31,1 & 0,256 \\
Mudança forçada de moradia & 148 & 42,7 & $<0,001$ \\
Rompimento de relação amorosa & 268 & 40,3 & $<0,001$ \\
Vítima de assalto ou roubo & 164 & 35,7 & $<0,001$ \\
Vítima de agressão física & 76 & 46,3 & $<0,001$ \\
\hline
\end{tabular}

mitem investigar a possibilidade de causalidade reversa. Entretanto, o fato da avaliação dos EVPE ter englobado os 12 meses anteriores ao preenchimento do questionário e a avaliação de TMC pelo GHQ-12 limitar-se aos 15 dias anteriores, minimiza esta possibilidade. Apenas uma parcela reduzida destes eventos poderá ter ocorrido durante o período avaliado pelo GHQ. Análises recém-iniciadas de dados colhidos em 2001 - Fase 2 do Estudo Pró-Saúde permitirão investigar a ocorrência de EVPE na "linha de base" como fatores de risco para manutenção e desenvolvimento de TMC após dois anos de seguimento, nesta população.

Outra limitação refere-se ao fato do GHQ-12 ser um instrumento de rastreamento para transtornos psiquiátricos menores, sendo sensível a mudanças recentes no estado psicológico dos indivíduos, e não permitindo a distinção daqueles com um diagnóstico psiquiátrico estabelecido. Tal fato pode levar à inclusão de falsos positivos no estudo, ou seja, de indivíduos com transtornos psicológicos transitórios, o que traria como conseqüência o aumento da prevalência de TMC nesta população. Entretanto, erros de classificação decorrentes dessa limitação tendem a ser não-diferenciais, o que resultaria em subestimação das associações. Além disso, o fato dos nossos resultados estarem próximos dos encontrados em outros estudos, sugere que o GHQ-12, com os pontos de corte adotados, é um instrumento adequado para ser usado na avaliação de prevalência de transtornos mentais comuns nesta população.

O fato da amostra deste estudo ser constituída por uma população de funcionários, ou seja, por população empregada e com estabili-

Tabela 4

Odds ratios (OR) brutas e ajustadas * e intervalos de $95 \%$ de confiança (IC 95\%) da associação entre eventos de vida produtores de estresse (EVPE) e transtornos mentais comuns (TMC), entre funcionários técnico-administrativos de uma universidade no Estado do Rio de J aneiro. Estudo Pró-Saúde, 1999.

\begin{tabular}{lcc}
\hline EVPE & OR bruta (IC95\%) & OR ajustada (IC95\%) \\
\hline Problema sério de saúde & $1,83(1,57-2,15)$ & $1,81(1,54-2,12)$ \\
Internação hospitalar & $1,45(1,13-1,86)$ & $1,37(1,06-1,77)$ \\
Dificuldades financeiras graves & $2,64(2,29-3,04)$ & $2,60(2,24-3,02)$ \\
Morte de parente próximo & $1,12(0,92-1,37)$ & $1,18(0,95-1,46)$ \\
Mudança forçada de moradia & $1,95(1,55-2,44)$ & $1,90(1,50-2,40)$ \\
Rompimento de relação amorosa & $1,84(1,55-2,19)$ & $1,94(1,61-2,34)$ \\
Vítima de assalto ou roubo & $1,42(1,16-1,74)$ & $1,46(1,18-1,80)$ \\
Vítima de agressão física & $2,19(1,60-3,01)$ & $2,13(1,54-2,95)$
\end{tabular}

* Ajustadas por sexo, idade, renda e situação conjugal. 
dade, diferencia-a da população geral, colocando-a em um patamar sócio-econômico mais elevado. Em populações submetidas a níveis sócio-econômicos mais baixos, à instabilidade no emprego, ou mesmo ao desemprego e, por conseguinte, mais sujeitas a eventos de vida estressantes, esses eventos tenderiam a assumir importância ainda maior na ocorrência de transtornos mentais comuns.

A possibilidade de viés do trabalhador saudável deve ser levada em consideração em estudos epidemiológicos conduzidos em populações empregadas. Em nosso estudo, como uma forma de minimizar este viés, foi feita busca ativa de todos os funcionários em licença médica. Eles foram localizados por telefone, e muitos compareceram à universidade para o preenchimento do questionário. Para a maioria dos funcionários que se achavam incapacitados de se locomover, foi realizada visita domiciliar.

\section{Agradecimentos}

À toda a equipe do Pró-Saúde, que participou das etapas do trabal ho de campo e digitação dos dados, e aos funcionários técnico-administrativos da universidade que responderam aos questionários desta pesquisa.

Este trabalho foi parcialmente financiado pelo Conselho Nacional de Desenvolvimento Científico e Tecnológico (Produtividade em Pesquisa - 300228/996) e Fundação de Amparo à Pesquisa do Estado do Rio de Janeiro (E-26/ 170.714/2001).

\section{Referências}

ARAYA, R.; WYNN, R. \& LEWIS, G., 1992. Comparison of two self-administered psychiatric questionnaires (GHQ-12 and SRQ-20) in primary care in Chile. Social Psychiatry and Psychiatric Epidemiology, 27:168-173.

BEBBINGTON, P. E.; HURRY, J.; TENNANT, C.; STURT, E. \& WING, J., 1981. Epidemiology of mental disorders in Camberwell. Psychological Medicine, 11:561-579.

BHUGRA, D., 1993. Unemployment, poverty and homelessness. In: Principles of Social Psychiatry (D. Bhugra \& J. Leff, ed.), pp. 355-382, Oxford: Blackwell Scientific Publications.

BROWN, G. W. \& HARRIS, T., 1978. Social Origins of Depression. A Study of Psychiatric Disorder in Women. Cambridge: University Press.
Os últimos anos, em nosso meio, têm sido marcados por uma conjuntura econômica desfavorável, com aumento da desigualdade social, pauperização crescente da classe média em geral e dos funcionários públicos de uma forma mais específica. Tal conjuntura, aliada a uma série de outros fatores como as altas taxas de violência e criminalidade, e o colapso na oferta de serviços nos setores públicos, contextualizam os nossos achados, reforçando a necessidade de disponibilização de serviços de apoio psicológico e social, de natureza preventiva, naqueles trabalhadores que estivessem passando por situações identificadas como de "risco".

Além disso, os resultados encontrados neste estudo apontam para a necessidade de se investigar efeitos de longo prazo associados à ocorrência de eventos de vida produtores de estresse, assim como de fatores que potencialmente modifiquem seu impacto (ex. apoio social).
BRUGHA, T. S.; BEBBINGTON, P.; STURT, E.; MaCCARTHY, B. \& WYKES, T., 1990. The relation between life events and social support networks in a clinically depressed cohort. Social Psychiatry and Psychiatric Epidemiology, 25:308-313.

BUSNELLO, E. D.; LIMA, B. R. \& BERTOLOTE, J. M., 1983. Aspectos interculturais de classificação e diagnóstico. Jornal Brasileiro de Psiquiatria, 32: 207-210.

CHENG, T. A., 1988. A community study of minor psychiatric morbidity in Taiwan. Psychological Medicine, 18:953-968.

CHENG, T. A., 1989. Urbanization and minor psychiatric morbidity. A community study in Taiwan. Social Psychiatry and Psychiatric Epidemiology, 24:309-316. 
COOKE, D. J., 1986. Psychosocial variables and the life event/anxiety-depression link. Acta Psychiatrica Scandinavica, 74:281-291.

COSTELLO, C. G., 1982. Social factors associated with depression: A retrospective community study. Psychological Medicine, 12:329-339.

COUTINHO, E. S. F., 1995. Fatores Sociodemográficos e Morbidade Psiquiátrica Menor: Homogeneidade e Heterogenei dade de Efeitos. Tese de Doutorado, Salvador: Universidade Federal da Bahia.

EATON, W. W., 1978. Life events, social supports, and psychiatric symptoms: A re-analysis of the $\mathrm{New}$ Haven data. Journal of Health and Social Behaviour, 19:230-234.

FAERSTEIN, E.; LOPES C. S.; VALENTE, K.; PLÁ, M. A. S. \& FERREIRA, B., 1999. Pré-testes de um questionário multidimensional autopreenchível: A experiência do Estudo Pró-Saúde. Physis, 9:117-130.

FINLAY-JONES, R. A. \& BROWN, G. W., 1981. Types of stressful life-events on the onset of anxiety and depressive disorders. Psychological Medicine, 11: 803-815.

FINLAY-JONES, R. A. \& BURVILL, P. W., 1977. The prevalence of minor psychiatric morbidity in the community. Psychological Medicine, 7:475-489.

FLEISS J. L., 1981. Statistical Methods for Rates and Proportions. 2nd Ed. New York: John Wiley \& Sons.

GOLDBERG, D. P. \& BLACKWELL, B., 1970. Psychiatric illness in general practice. A detailed study using a new method of case identification. BMJ, 1:439-443.

GOLDBERG, D. P. \& BLACKWELL, B., 1972. The Detection of Psychiatric Illness by Questionnaire. Maudsley Monograph 21. London: Oxford University Press.

GOLDBERG, D. P. \& HUXLEY, P., 1992. Common Mental Disorders. A Bio-Social Model. London: Routledge.

GOLDBERG, D. P. \& WILLIAMS, P., 1988. The User's Guide to the General Health Questionnaire. Windsor: Nfer-Nelson.

HOROWITZ, M.; SCHAEFER, C.; HIROTO, D.; WILNER, N. \& LEVIN, B., 1977. Life event questionnaires for measuring presumptive stress. Psychosomatic Medicine, 39:413-431.

IACOPONI, E., 1989. The Detection of Emotional Disorders by Primary Care Physi cians: A Study in São Paulo, Brazil. Ph.D. Thesis, London: University of London.

KENDLER, K. S.; KARKOWSKI, L. M. \& PRESCOTT, C. A., 1998. Stressful life events and major depression: Risk period, long-term contextual threat, and diagnostic specificity. Journal of Nervous and Mental Disease, 186:661-669.

KENDLER, K. S.; KESSLER, R. C.; WALTERS, E. E.; MACLEAN, C. .J.; SHAM, P. C.; NEALE, M. C.; HEATH, A. C. \& EAVES, L. J., 1995. Stressful life events, genetic liability and onset of an episode of major depression in women. American Journal of Psychiatry, 152:833-842.

LANDIS, J. R. \& KOCH, G. G., 1977. The measurement of observer agreement for categorical data. Biometrics, 33:159-174.

LIMA, M. S.; BERIA, J. U.; TOMASI, E.; CONCEIÇÃO, A. T. \& MARI, J. J., 1996. Stressful life events and minor psychiatric disorders: An estimate of the population attributable fraction in a Brazilian community-based study. International Journal of Psychiatry in Medicine, 26:211-222.

LOPES, C. S. \& FAERSTEIN, E., 2001. Confiabilidade do relato de eventos de vida produtores de estresse em um questionário multidimensional autopreenchido em uma amostra de funcionários de uma universidade pública: Estudo Pró-Saúde. Revista Brasileira de Psiquiatria, 23:126-133.

MARI, J. J., 1986. Minor Psychiatric Morbidity in Three Primary Care Clinics in the City of São Paulo. Ph.D. Thesis, London: Institute of Psychiatry, University of London.

MARI, J. J., 1987. Psychiatric morbidity in three primary medical care clinics in the city of São Paulo. Issues on the mental health of the urban poor. Social Psychiatry, 22:129-138.

MARI, J. J. \& WILLIAM S, P., 1985. A comparison of the validity of two psychiatric screening questionnaires (GHQ-12 and SRQ-20) in Brazil, using Relative Operating Characteristic (ROC) analysis. Psychological Medicine, 15:651-659.

PAYKEL, E. S., 1978. Contribution of life events to causation of psychiatric illness. Psychological Medicine, 8:245-253.

SARTORIUS, N.; JABLENSKY, A.; KORTEN, A.; ERNBERG, G.; ANKER, M.; COOPER, J. E. \& DAY, R., 1986. Early manifestations of first-contact incidence of schizophrenia in different cultures: A preliminary report on the initial evaluation phase of the WHO Collaborative Study of Determinants of Outcome of Severe Mental Disorders. Psychological Medicine, 16:909-928.

STANFELD, S. A. \& MARM OT, M. G., 1992. Social class and minor psychiatric morbidity. A validated screening survey using the General Health Questionnaire in British civil servants. Psychological Medicine, 22:739-749.

STANFELD, S. A.; RAEL, E. G.; SHIPLEY, M \& \& MARMOT, M., 1997. Social support and psychiatric sickness absence: A prospective study of British civil servants. Psychological Medicine, 27:35-48.

SURTEES, P. G.; MILLER, P. M.; INGHAM , J. G.; KREITMAN, N. B.; RENNIE, D. \& SASHIDARAN, S. P., 1986. Life events and the onset of affective disorder: A longitudinal general population study. Journal of Affective Disorders, 10:37-50.

VILLANO, L. A. B., 1998. Problemas Psicológicos e Morbidade Psiquiátrica em Serviços de Saúde Não-Psiquiátricos: O Ambulatório de Clínica Geral. Tese de Doutorado, São Paulo: Escola Paulista de Medicina.

WEICH, S. \& LEWIS, G., 1998. Poverty, unemployment, and common mental disorders: Population based cohort study. BMJ, 317:115-119.

WHO (World Health Organization), 1979. Schizophre nia. International Follow-up Study. New York: John Wiley.

Recebido em 23 de julho de 2002

Versão final reapresentada em 6 de maio de 2003

Aprovado em 31 de julho de 2003 